\title{
Eğirdir İlçesi (Isparta) İçin Kaya Düşmesi Duyarlılık Bölgelerinin Haritalandırılması
}

\author{
Kerem Hepdeniz ${ }^{1 *}$ \\ ${ }^{1}$ Mehmet Akif Ersoy Üniversitesi, Bucak Emin Gülmez T.B.M.Y.O, Mimarlık ve Şehir Planlama Bölümü, Burdur, Türkiye (ORCID: 0000-0003-4182-5570)
}

(İlk Geliş Tarihi 6 Şubat 2019 ve Kabul Tarihi 2 Mart 2019)

(DOI: 10.31590/ejosat.523612)

ATIF/REFERENCE: Hepdeniz, K. (2019). Eğirdir İlçesi (Isparta) İçin Kaya Düşmesi Duyarllılı Bölgelerinin Haritalandırılması. Avrupa Bilim ve Teknoloji Dergisi, (15), 193-198.

\section{$\ddot{O} \mathbf{z}$}

Türkiye, bulunduğu coğrafi konum ve atmosfer koşulları nedeniyle, doğal afetlerin çok sık görüldüğü bir ülkedir. Bu doğal afetler her yıl çok sayıda can ve mal kaybına neden olmaktadır. Ancak bu doğal afetlerin çoğunun önceden tahmin edilmesi mümkün değildir. Can ve mal kaybının önüne geçilmesi için ilk önce yapılması gereken; bu doğa olaylarını iyi tanımak, risk taşıyan alanları önceden tespit etmek ve zararların azaltılabilmesi için gerekli önlemleri almak olmakdır. Kaya düşmeleri, diğer afet türlerine göre daha küçük ölçekteki bölgesel alanları etkilemekte ancak meydana geldiği yer açısından yıkıcı sonuçlar oluşturmaktadır. Ancak maalesef ülkemizde, yerleşim ve konut alanlarının yer seçiminde afet açısından kapsamlı bir inceleme ve alt yapı çalışmalarının yapılmadığı görülmektedir. Bu çalışmada, Coğrafi Bilgi Sistemleri, Conefall ve Google Earth programları kullanılarak, Isparta İli Eğirdir İlçe merkezi için kaya düşmesi duyarlılık haritalarının hazırlanması amaçlanmıştır. Çalışma alanına ait 1/25000 ölçekli topografik haritaların sayısallaştırılması ile elde edilen $5 \times 5$ metre çözünürlüğe sahip sayısal yükseklik modeli haritası ile kaya düşmesi için potansiyel olabilecek kaynak alanlar belirlenmiş ve Conefall programı ile yüksek-orta ve düşük duyarlılığa sahip alanlar haritalandırılmıştır. Bu haritalar gerekli format değişikliğinin ardından Google Earth programına aktarılmış; söz konusu alanda yüksek-orta ve düşük duyarlılık bölgesi içerisinde kalan binaların olduğu; Isparta' yı Eğirdir ve Konya' ya bağlayan D-350 karayolunun bazı kesimlerinin de yine yüksek-orta ve düşük derece duyarlılık bölgesinde kaldığı görülmüştür. Yapılan arazi gözlemleri ile yüksek ve orta derecede duyarlı alanlarda daha önceden düşen kaya blokları gözlemlenmiş, genel kayaç yapısının ise süreksizlik düzlemlerine sahip kireçtaşı bloklarından oluştuğu tespit edilmiş̧ir. Bu bölgelerde meydana gelebilecek kaya düşmesi durumunda olası can ve mal kaybının önüne geçilebilmesi ancak gerekli tedbirlerin alınması ile mümkün olabilecektir. Bölgedeki yerel yönetim ve birimlerin söz konusu alan ile ilgili olarak gerekli önlemleri almalarının son derece önemli olduğu düşünülmektedir.

\section{Mapping of Rockfall Susceptibility Areas for Eğirdir District (Isparta)}

\begin{abstract}
Natural disasters are very common in Turkey, due to its geographic location and atmospheric condition. These natural disasters cause a lot of life and property loss every year. However, it is not possible to predict many of these natural disasters. In order to prevent loss of life and property should be done first, to recognize these natural phenomena well, to identify the risk areas in advance and to take necessary measures to reduce the damage should be. Rock falls affect more regional areas than other types of disasters, but they have devastating consequences for the place where they occur. Unfortunately, in our country, it is observed that a comprehensive investigation and infrastructure works have not been done in terms of disaster in the location of residential areas and housing zones. In this study, it is aimed to prepare rockfall susceptibility maps for Eğirdir district of Isparta province by using Geographic Information Systems,
\end{abstract}

\footnotetext{
${ }^{1}$ Sorumlu Yazar: Mehmet Akif Ersoy Üniversitesi, Bucak Emin Gülmez T.B.M.Y.O, Mimarlık ve Şehir Planlama Bölümü, Burdur, Türkiye ORCID: 0000-0003-4182-5570, khepdeniz@,mehmetakif.edu.tr
} 
Conefall and Google Earth programs. With a resolution of $5 \times 5$ meter digital elevation model map obtained by digitization of the $1 / 25000$ topographic maps of the study area, potential areas for potential rock fall were determined and with the help of Conefall program, high medium and low susceptibility area were mapped. These maps were transferred to Google Earth after the format changes, and some buildings and the some part of the D-350 highway; connecting Isparta to Eğirdir and Konya have been seen in the high and medium susceptibility zone. In high and medium susceptibility areas, rock blocks which had been fallen earlier were observed and it was determined that the general rock structure consists of limestone blocks with discontinuity surface. It is possible to prevent possible loss of life and property in case of rock fall in these regions, but it will be possible to take necessary measures. It is considered that it is important for the local authorities and the units in the region to take necessary measures regarding the area.

Keywords: Rock fall, Geographic information systems, Eğirdir, Conefall, Susceptibility map

\section{Giriş}

Kaya düşmesi, birkaç $\mathrm{dm}^{3}$ ' ten $10.000 \mathrm{dm}^{3}$ ' e kadar olan blokların eğim yönünde hareket etmesi olarak tanımlanır ve kayma, devrilme veya düşme şeklinde gerçekleşir (Dussauge-Peisser, ve diğerleri, 2002) (Aksoy \& Ercanoğlu, 2006) (Loye, Jaboyedoff, \& Pedrazzini, 2009). Bu blokların hacmi ise kaynak alanlarındaki süreksizliklerin set sayısı, yönelimi ve aralığı gibi süreksizlik özelliklerine bağlıdır (T.C. Başbakanlık Afet ve Acil Durum Yönetimi Başkanlığı, 2015).

Önceden tahmin edilmesi oldukça güç olan kaya düşmesi, farklı jeolojik, jeomorfolojik, iklimsel (donma ve çözülme, gözenek basıncı, kimyasal ve fiziksel ayrışma) ve insan etkisi gibi faktörlere bağlıdır (Aksoy \& Ercanoğlu, 2006). Dağlık alanlardaki kaya düşmesi riski, nüfus ve ekonomik aktivitenin artması ile paralellik gösterir (Baillifard, ve diğerleri, 2004) (Leroi, Bonnard, Fell, \& Innes Mc, 2005) ve kaya düşmeleri özellikle yerleşim yerleri ve otoyollara yakın kesimlerde meydana geldiğinde risk oluşturur. Ülkemizin iklim, jeolojik ve jeomorfolojik özelliklerinin her türlü yamaç hareketi için uygun olmasının yanısıra, yerleşim ve konut alanlarının yer seçiminde gerekli hassasiyetin gösterilmemesi, görülen zararları daha da arttırmaktadır (Şahin \& Sipahioğlu, 2009).

Tüm kütle hareketlerinin zararlarını azaltmak için önerilen yöntemler, önleyici ve koruyucu türdendir. Kaya düşmesi tehlike haritalarının üretilmesinde, aktif kaynak alanlarının mevcudiyeti, en büyük menzil zonunun belirlenmesi, bu zonda evler, yollar, elektrik hatları ve alt yapı gibi beşeri faktörlerin olup olmadığı ve kaynak alanlar ile insan yaşam alanları arasında ağaçlık alanların mevcudiyeti gibi sorulara cevap verilmelidir (Larcher, Simoni, Pasquazzo, Strada, \& Zampedri, 2012).

Bu çalışmada, Isparta' ya bağlı Eğirdir ilçe merkezi ve civarının kaya düşmesi duyarlılık haritalarının oluşturulması için Coğrafi Bilgi Sistemleri ArcGIS 10 programı kullanılarak potansiyel kaya düşmesi kaynak alanları tespit edilmiştir. Bu alanlar Conefall yazılımından faydalanılarak yüksek, orta ve düşük kaya düşmesi potansiyeli olan alanlara ayrılarak, gerekli dönüşüm işlemlerinden sonra Google Earth programına aktarılmıştır. Yapılan duyarlılık haritaları sonucunda yüksek ve orta kaya düşmesi potansiyeli olan alanlar saha çalışmaları ile de gözlemlenmiş ve kayaçların yapısı ve jeolojik özellikleri not edilmiştir.

\section{2. Çalıșma Alanı}

Isparta il sınırları içerisinde yer alan Eğirdir ilçesinin yüzölçümü $1414 \mathrm{~km}^{2}$, deniz seviyesinden yüksekliği ise 918 metredir. Kuzeyden Yalvaç ve Gelendost, doğudan Şarkikaraağaç ve Aksu, güneyden Sütçüler, güneybatıdan Burdur ili, batıdan Isparta merkez ve Atabey ile kuzeybatıdan Senirkent ilçeleri ile komşudur (Şekil 1). İlçenin yüzey şeklini, Eğirdir Gölü ile Isparta çöküntü alanını ayıran dağlar oluşturur. Genel olarak Akdeniz ve karasal iklimin hâkim olduğu ilçede kışlar soğuk ve yağışlı, yazlar ise sıcak ve kuraktır (Uysal, 2011; Dağıstanlıŏglu, 2012). 


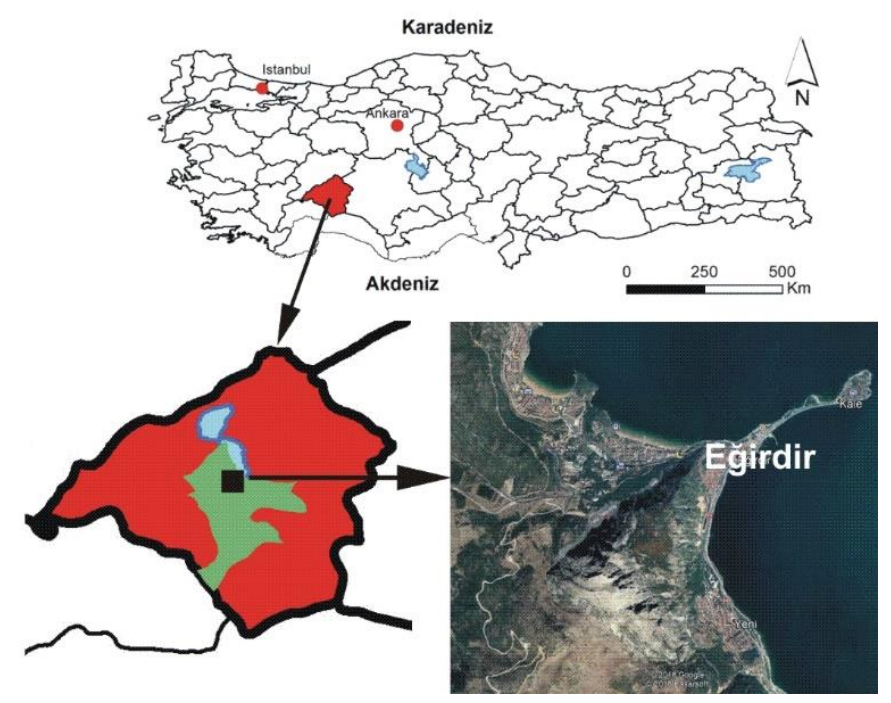

Şekil 1: Eğirdir ilçe merkezi yerbulduru haritası

\section{Materyal ve Metod}

Kaya düşmesine neden olabilecek aktif kaynak alanların saha çalışmaları ile belirlenmesi oldukça zor ve zaman gerektiren bir husustur. Kaya düşmesi olayının meydana gelmesi için temel faktörlerden biri dik eğim açısı olduğu bilinmektedir (Heim, 1932). Bu nedenle Coğrafi Bilgi Sistemleri programı yardımıyla elde edilecek sayısal yükseklik modeli (DEM) haritaları ile potansiyel kaynak alanlarını tespit etmek mümkün olmaktadır. Buradaki önemli husus elde edilecek DEM haritasının çözünürlüğüdür. Çözünürlüğün yüksek olması, modellenen arazinin eğim açılarının gerçeğe çok yakın olmasını sağlamaktadır (Loye, Jaboyedoff, \& Pedrazzini, 2009). $\mathrm{Bu}$ çalışmada, çalışma alanını kapsayan 1/25000 ölçeğindeki M25b1 ve M25b4 paftaları ArcGIS 10 programı kullanılarak sayısallaştırılmış ve $5 \mathrm{~m}$ çözünürlüğe sahip DEM haritası elde edilmiştir. Olası kaynak alanlarının Coğrafi Bilgi Sistemleri temelli belirlenmesinde ise aşağıdaki formül kullanılmıştır (Dorren \& Seıjmonsbergen, 2003; Troisi, Berger, \& Dorren, 2008).

$\alpha=55 *$ RES-0.075

$\alpha$ (derece): potansiyel kaynak alanları için sınır eğim değeri

RES: sayısal arazi modelinin çözünürlüğü

Yukarıdaki formüle göre 5 m çözünürlüğe sahip DEM haritası için kritik eğim açısı değeri $49^{\circ}$ olarak bulunmuştur. Elde edilen sonuca göre, $49^{\circ}$ ve üzerindeki eğim değerine sahip alanlar, kaya düşmesinin gerçekleşebileceği potansiyel kaynak alanları oluşturmaktadır. DEM haritasından elde edilen eğim haritası, $49^{\circ}$ lik eşik eğim açısı dikkate alınarak yeniden sınıflandırılmış (reclassify) ve Conefall programına kullanımını uygun hale getirmek için $49^{\circ}$ ' nin üzerindeki eğim açıları için “+1”; diğer eğim değerleri için "-1" değeri atanmıştır (Şekil: 2). 


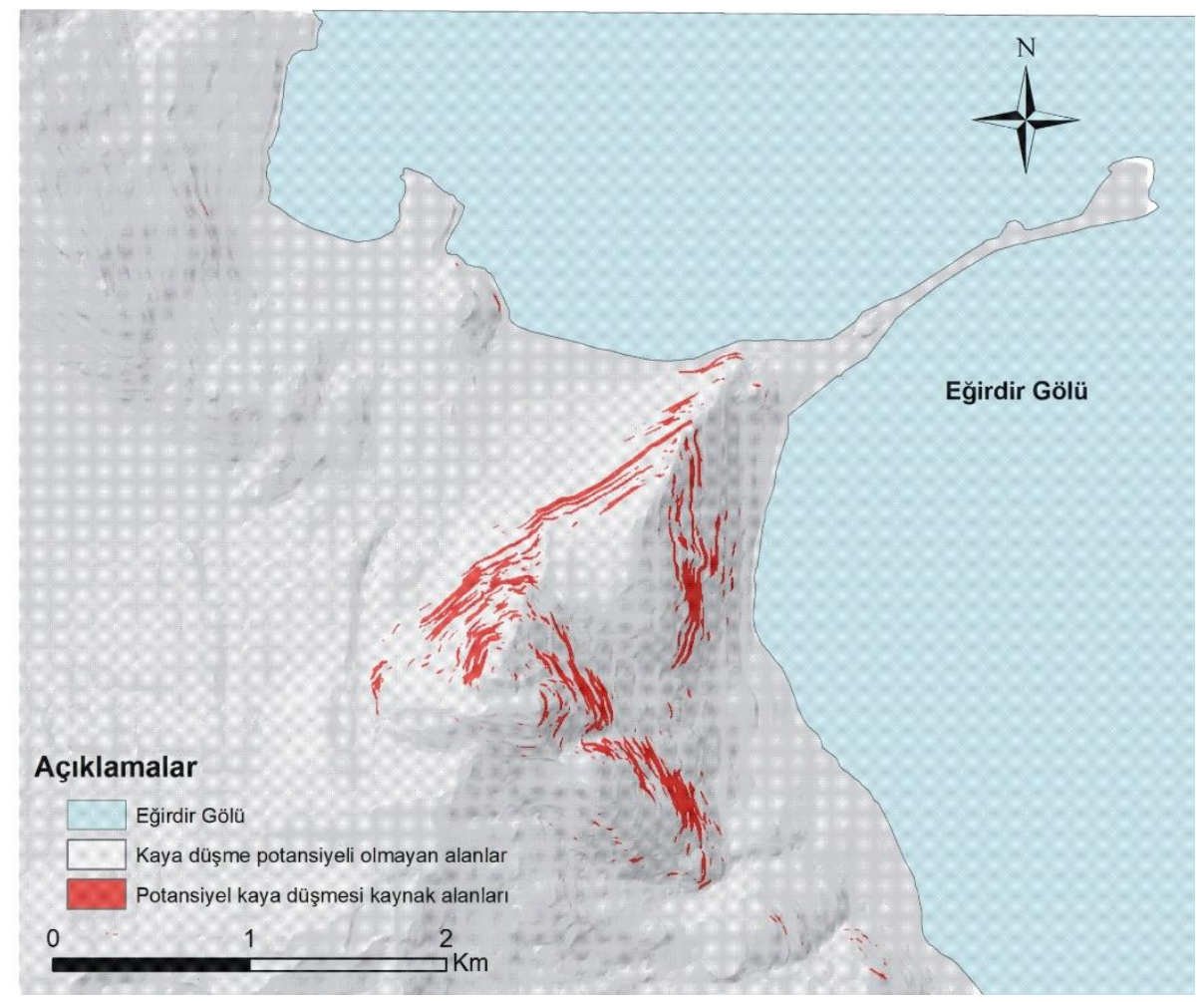

Şekil 2: Eğirdir ilçe merkezi potansiyel kaya düşmesi kaynak alanları haritası

Haritada kırmızı renk ile görülen alanlar, potansiyel kaya düşmesi kaynak alanlarını gösterirken; gri renkli bölgeler kaya düşmesi için kaynak olamayacak alanlara karşıllık gelmektedir. Bu aşamadan sonra ise elde edilen haritanın, Conefall yazılımında kullanılabilmesi için ArcGIS 10 programı içerisinde raster formatından ASCII formatına dönüştürülmüştür.

Kaya düşmesi yayılım alanları, Conefall bilgisayar programında uygulanan basit bir Coulomb sürtünme modeline dayanan gölge açısı veya enerji hattı yöntemi olarak bilinen basit bir geometrik kural kullanılarak belirlenebilir (Jaboyedoff \& Labiouse, 2011) ve topografik özellikler, DEM haritası, kaya düşmesi kaynak alanları ve konik yayılım açısı kullanılır. Gölge açısı değeri, eğimin azaldığı kesimden, kaya bloğunun ulaşabildiği en uzak nokta arasındaki enerji çizgisinin açı değeri olarak ifade edilmektedir (Capons, Vilaplana, \& Linares, 2009). Önceki çalışmalar, gölge açısının alabileceği değerleri, yapılan varsayımlara bağlı olarak $22^{\circ}$ ile $38^{\circ}$ arasında değiştiğini göstermektedir (Toppe, 1987; Evans \& Hungr, 1993; Wieczorek, Morrissey, lovine, \& Godt, 1999). Yapılan bu çalışmada ise kaya düşmesi duyarlılık haritalarının belirlenmesinde gölge açısı sınır değerleri, literatür çalışmaları da dikkate alınarak $32^{\circ}$ (düşük), $35^{\circ}$ (orta) ve $38^{\circ}$ (yüksek) değerleri verilerek duyarlılık sınıfları oluşturulmuştur. Conefall programı içerisinde oluşturulan bu sınıf değerlerinin ArcGIS 10 programı içerisinde kullanılabilmesi için tekrar dönüşüm işlemi uygulanarak raster formatına çevrilmiştir. Kaya düşmesi için elde edilen 3 farklı seviyedeki duyarlılık haritaları aritmetik olarak toplanmıştır (Şekil: 3). 


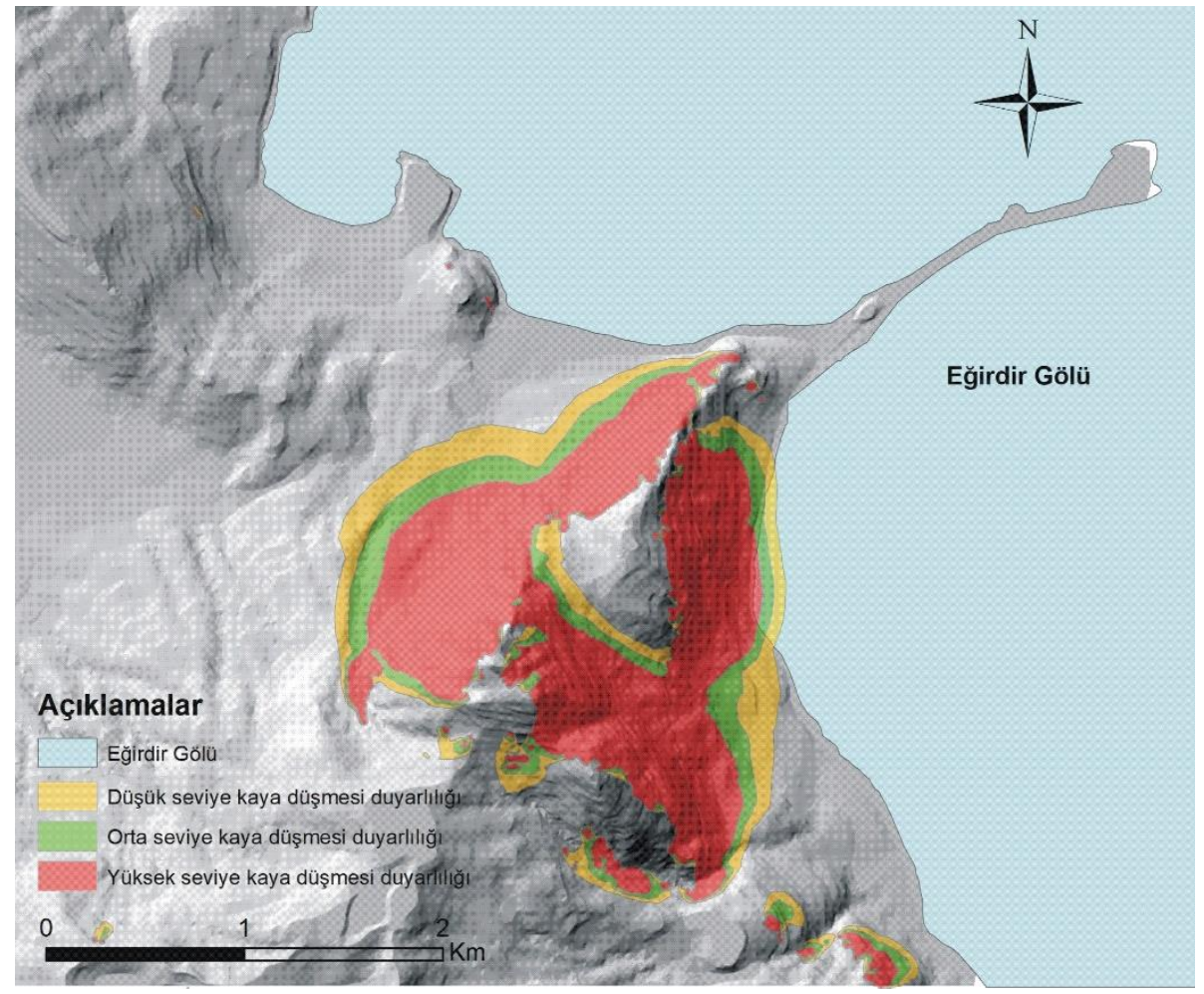

Şekil 3: Eğirdir ilçe merkezi kaya düşmesi duyarlılık zonları haritası

Elde edilen haritada; sarı renk ile gösterilen alanların düşük, yeşil renk ile gösterilen alanların orta ve kırmızı renk ile gösterilen alanların ise yüksek kaya düşmesi duyarlılığına sahip alanlar olduğu belirtilmektedir.

$\mathrm{Bu}$ işlemlerin ardından raster formatındaki duyarlılık haritası $\mathrm{Kml}$ formatına dönüştürülmüşs ve kaya düşmesi duyarlılık haritası Google Earth programına aktarılarak yüksek-orta ve düşük kaya düşmesi duyarlılık zonu içerisinde kalan yerleşim yerleri, binalar ve yollar görüntülenebilmiştir (Şekil 4).
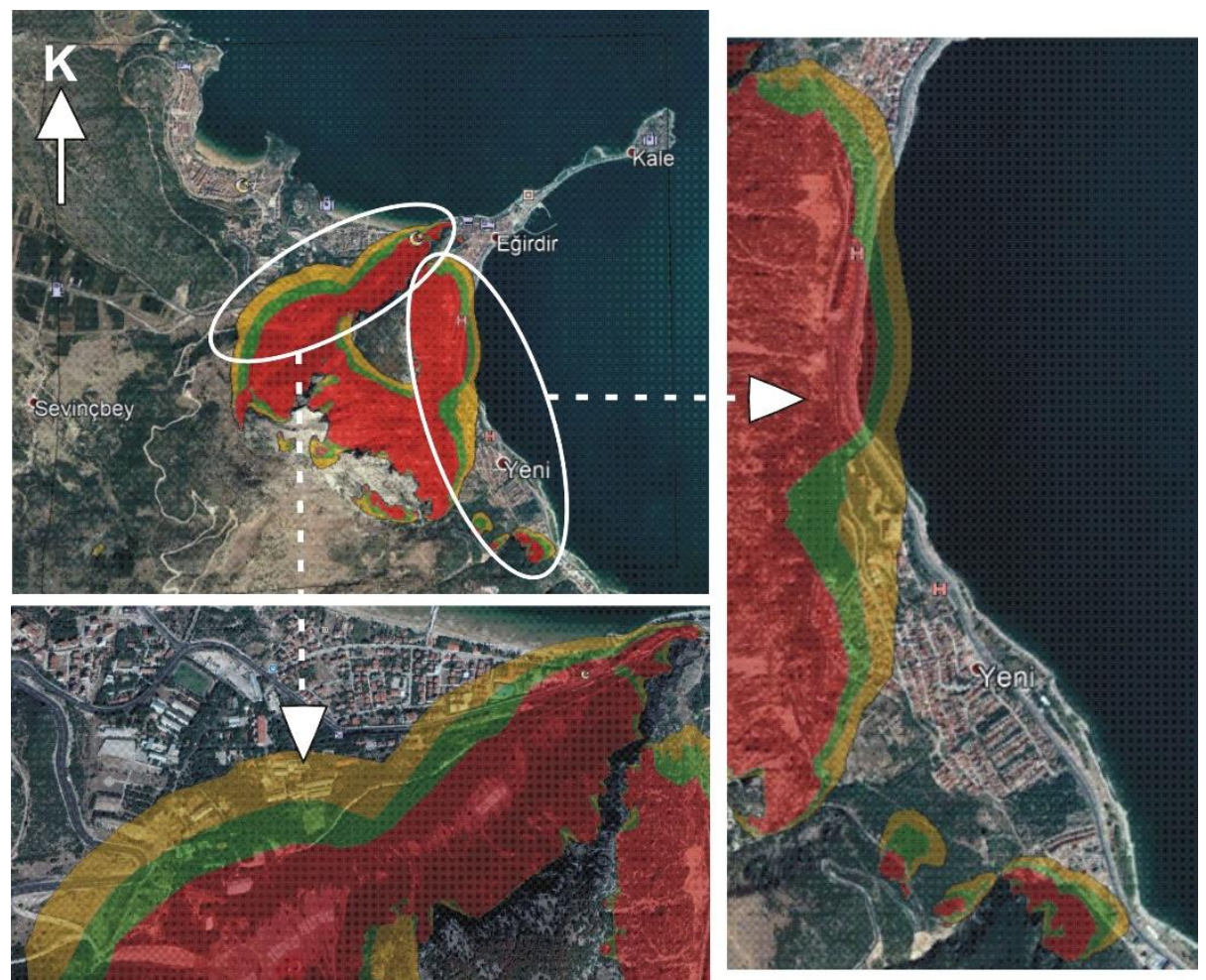

Şekil 4: Google Earth programına aktarılan duyarlılık zonları

\subsection{Kaya Düşmesi Duyarlıık Haritalarının Değerlendirilmesi ve Arazi Gözlemleri}

Google Earth görüntüsü ve arazi gözlemleri sonucunda çalışma alanı içerisinde yüksek, orta ve düşük kaya düşmesi duyarlılığına sahip alanların mevcut olduğu ortaya çıkmıştır. Çalışma alanı doğu kesimi; Eğirdir-Konya karayolu üzerinde bulunan bazı binaların ve 
Eğirdir' i Konya' ya bağlayan karayolunun bir kısmının yüksek ve orta duyarlılık alanı içerisinde olduğu (Şekil 5 A-B), çalışma alanı kuzey ve kuzeybatı kesimlerinde ise yine yüksek ve orta duyarlılık zonunda bulunan binaların olduğu görülmüştür (Şekil 5 E). Çalışma alanı doğu kesiminde yer alan binaların dağ yamacına bakan batı kesiminde eğim atımlı bir fay aynası mevcuttur (Şekil 5 C). Bölgenin 1. Derece deprem riski altında bulunması bölgeyi daha duyarlı hale getirmektedir. Ayrıca, bu bölgede yer alan kireçtaşlarının çok sayıda kırık çatlak içerdiği ve birçok kaya bloğununda yuvarlanarak binaların hemen arkasında yol seviyesine kadar indiği gözlemlenmiştir (Şekil 5 D-F). Yamaçlarda ise küçük tetiklenmeler ile düşmeye hazır birçok gevşek blokta mevcuttur.
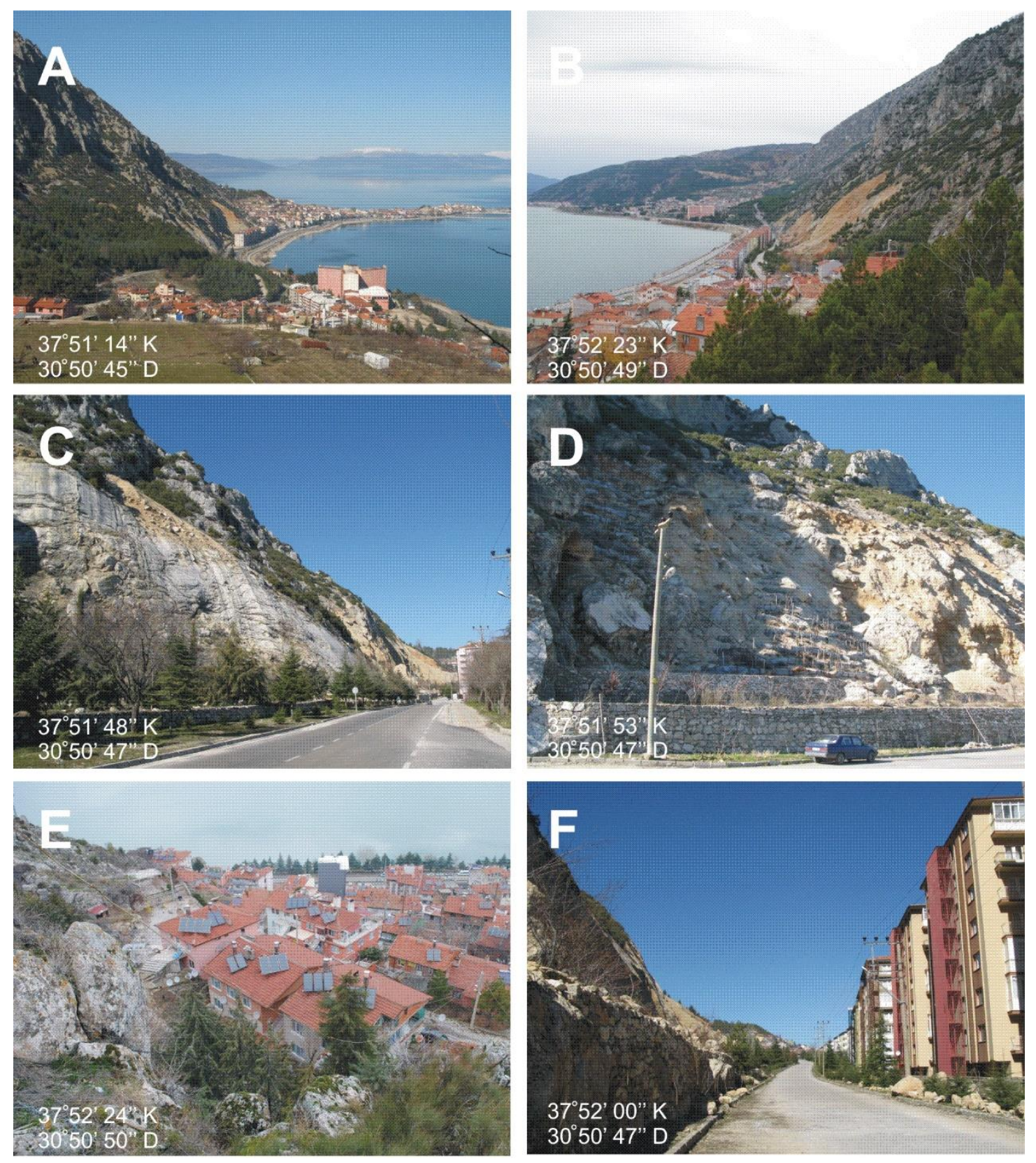

Şekil 5: Eğirdir ilçe merkezi ve civarından çekilen fotoğraflar ve koordinat bilgileri

\section{Sonuç}

Son yıllarda, CBS teknolojileri afet yönetimi, duyarlılık haritalarının hazırlanması ve tehlike azaltma konularında sıklıkla kullanılmakta ve mekânsal planlama ile sürdürülebilir kalkınma alanlarında giderek daha önemli bir rol oynamaktadır. Kaya düşmeleri, diğer kütle hareketlerine kıyasla, daha küçük alanlarda etkili olsalar da, oldukça yıkıcı etkilere sahiptir. Bu amaçla, Eğirdir ilçe merkezi için yapılan çalışmada, kaya düşmesi duyarlılık analizi haritalaması sonucunda, bazı binaların yüksek-orta duyarlılık alanı içerisinde olduğu tespit edilmiş ve elde edilen sonuç, arazi gözlemleri ile de desteklenmiştir. Arazi gözlemleri sırasında bölgede daha önceden düşen kaya bloklarının varlığı belirlenmiştir. Arazinin genel jeolojik yapısını oluşturan kireçtaşı bloklarının kırık çatlak sistemine sahip olması, bölgenin 1. derece deprem risk alanı içerisinde yer alması ve kış aylarında donma çözünme gibi iklimsel bir yapıda olması, sahayı kaya düşmesi açısından daha riskli hale getirmektedir. Olası bir kaya düşmesi sonucunda meydana gelebilecek can ve mal kayıplarının önüne geçilebilmesi ve risklerin en düşük seviyeye indirilebilmesi için, söz konusu orta ve yüksek duyarlılığa sahip alanlarda yerel yönetimler ve yetkililer tarafından gereken iyileştirici ve önleyici tedbirlerin alınmasının önemli olduğu düşünülmektedir. 


\section{Kaynakça}

Aksoy, H., \& Ercanoğlu, M. (2006). Determination of the rockfall source in an urban settlement area by using a rule-based fuzzy evaluation. Natural Hazards and Earth System Sciences, 941-954.

Baillifard, F., Jaboyedoff, M., Rouiller, J. D., Couture, R., Locat, J., Robichaud, G., \& Gamel, G. (2004). Towards a GIS-based hazard assessment along the Quebec City Promontory,Quebec,Canada. W. Lacerda, M. Ehrlich, A. Fontoura, \& A. Sayao içinde, Landslide Evaluation and Stabilization (s. 207-213). Canada.

Capons, R., Vilaplana, J., \& Linares, R. (2009). Rockfall travel distance analysis by using empirical models (Sola d'Andorra la Vella, Central Pyrenees). Natural Hazards Earth System Sciences, 2107-2118.

Dağıstanlığlu, C. (2012). Eğirdir Katı Atık Deponi Alanının Yer Seçimi Kriterlerinin Coğrafi Bilgi Sistemleri (CBS) İle Belirlenmesi. İzmir: Ege Üniversitesi Fen Bilimleri Enstitüsü.

Dorren, L., \& Seijmonsbergen, A. (2003). Comparisonof three GIS-based models for predicting rockfall runout zones at a regional scale. Geomorphology, 49-64.

Dussauge-Peisser, C., Helmstetter, A., Grasso, J. R., Hantz, D., Desvarreux, P., Jeannin, M., \& Giraud, A. (2002). Probabilistic approach to rock fall hazard assessment: potential of historical data analysis. Natural Hazards and Earth System Sciences, 15-26.

Evans, S., \& Hungr, O. (1993). The assessment of rockfall hazard at the base of talus slopes. Canadian Geotechnical Journal, $620-636$.

Heim, A. (1932). Bergsturz und Menschenleben, Fretz and Wasmuth Verlag. Zurich.

Jaboyedoff, M., \& Labiouse, V. (2011). Technical Note: Preliminary estimation of rockfall runout zones. Natural Hazards and Earth System Sciences, 819-828.

Larcher, V., Simoni, S., Pasquazzo, R., Strada, C., \& Zampedri, G. (2012). WP6 Guidelines Rockfall and Forecast Systems. Paramount.

Leroi, E., Bonnard, C., Fell, R., \& Innes Mc, R. (2005). Risk assessment and management. O. Hungr, R. Fell, R. Couture, \& E. Eberhardt içinde, Landslide Risk Management (s. 159-198). CRC Press.

Loye, A., Jaboyedoff, M., \& Pedrazzini, A. (2009). Identification of potential rockfall source areas at a regional scale using DEM-based geomorphometric analysis. Natural Hazards and Earth System Sciences, 1643-1653.

Şahin, C., \& Sipahioğlu, Ş. (2009). Doğal Afetler ve Türkiye. Ankara: Gündüz Eğitim ve Yayıncılık.

T.C. Başbakanlık Afet ve Acil Durum Yönetimi Başkanlığı. (2015). Bütünleşik Tehlike Haritalarının Hazırlanması Heyelan-Kaya Düşmesi Temel Kılavuz. Ankara: AFAD.

Toppe, R. (1987). Terrain models: A tool for natural hazard mapping. International Association of Hydrological Sciences, Wallingford, $U K, 629-638$.

Troisi, C., Berger, F., \& Dorren, L. (2008). Protection de la viabilite alpine. PROVIALP project report.

Uysal, K. (2011). Eğirdir-Burdur Gölleri Çevrelerindeki Pliyo-Kuvaterner Çökellerinin Stratigrafik, Sedimantolojik ve Bazı Tektonik Özellikleri. Isparta: Süleyman Demirel Üniversitesi Fen Bilimleri Enstitüsü.

Wieczorek, G., Morrissey, M., lovine, G., \& Godt, J. (1999). Rockfall potential in the Yosemite Valley, California. California: USGS Open file. 